\title{
Avaliação de um Modelo de Intervenção Psicológica para Meninas Vítimas de Abuso Sexual ${ }^{1}$
}

\author{
Luísa F. Habigzang ${ }^{2}$ \\ Roberta Hatzenberger \\ Fabiana Dala Corte \\ Fernanda Stroeher \\ Sílvia Koller \\ Universidade Federal do Rio Grande do Sul
}

\begin{abstract}
RESUMO - O abuso sexual contra crianças e adolescentes é considerado um importante problema de saúde pública. Dessa forma, é necessário o desenvolvimento de estudos que avaliem a efetividade de modelos de intervenção psicológica. O presente estudo tem como objetivo avaliar um modelo de grupoterapia cognitivo-comportamental para meninas vítimas de abuso sexual. Participaram 10 meninas com idade entre 9 e 13 anos, vítimas de pelo menos um episódio de abuso sexual intrafamiliar. O tipo de abuso sexual variou entre os casos, sendo que, em sete, ocorreram manipulação de genitais e assédio e, em três, relações sexuais com penetração. O delineamento utilizado foi medidas repetidas, realizadas antes, durante e depois da intervenção. Foram avaliados sintomas de depressão, ansiedade, estresse e transtorno do estresse pós-traumático. O modelo avaliado é constituído por 20 sessões com atividades semi-estruturadas. Os resultados apontaram a redução significativa dos sintomas e a reestruturação de crenças disfuncionais relacionadas ao abuso.
\end{abstract}

Palavras-chave: abuso sexual; terapia cognitivo-comportamental; avaliação; infância; adolescência.

\section{Assessment of a Psychology Intervention Model Effect on Sexual Abused Girls}

\begin{abstract}
The sexual abuse against children and adolescents is an important public health problem. In this way, it is necessary to develop studies that evaluate the models effectiveness of psychological intervention. The present study aimed to evaluate a group therapy, based on cognitive-behavioral approach to the girls who are victims of sexual abuse. Ten girls from 9 to 13 years old intrafamilial victimized by sexual abuse participated in this study. The type of sexual abuse varied among the cases: manipulation of genital and sexual harassment appeared in seven cases, and complete intercourse with penetration on the other three. The group therapy model evaluated was constituted by 20 sessions with semi-structured activities. Repeated measures were used to analyze the before, during and after intervention data on depression, anxiety, stress, and posttraumatic stress symptoms. A significant reduction of those aspects was found, as well as the restructuring of faiths related to the abuse.
\end{abstract}

Key words: sexual abuse; cognitive-behavioral therapy; evaluation; childhood; adolescence.

O abuso sexual contra crianças e adolescentes tem sido considerado um importante problema de saúde pública devido aos altos índices de incidência e às consequiências negativas que essa forma de violência pode ocasionar ao desenvolvimento psicológico e social das vítimas (Habigzang \& Caminha, 2004; Jonzon \& Lindblad, 2004; Osofsky, 1995). A intervenção terapêutica em casos de abuso sexual é complexa e precisa ser planejada, considerando o impacto dessa experiência para o desenvolvimento da vítima e da sua família, mudanças no ambiente imediato, disponibilidade de rede de apoio social e afetiva e fatores de risco e proteção associados (Cohen \& Mannarino, 2000; Saywitz, Mannarino, Berliner \& Cohen, 2000).

Uma meta-análise sobre pesquisas publicadas em língua inglesa que avaliaram formas de tratamento psicológico para vítimas de abuso sexual no período entre 1975 e 2004 identificou apenas 28 estudos controlados na área (Hetzel-Riggin, Brausch \& Montgomery, 2007). Poucos estudos controlados têm sido reali-

1 Financiamento: Edital MCT-CNPq/MS-SCTIE-DECIT/CT-Saúde-№ 07/2005.

2 Endereço: Rua Ramiro Barcelos, no 2600, sala 104, Porto Alegre, RS, Brasil 90035-003. E-mail: luisa.h@terra.com.br zados para avaliar os resultados de tratamentos, por uma série de obstáculos: dificuldade em identificar sintomas devido à falta de capacidade das crianças em descrever alterações comportamentais, afetivas e cognitivas; dificuldade em obter amostras homogêneas, em função de diferenças, tais como idade, gênero, nível socioeconômico, tempo de exposição ao abuso e gravidade do abuso, que impossibilitam uma padronização de procedimentos; e dificuldade em controlar a interferência de outras experiências de vida da criança, uma vez que o abuso sexual é apenas uma parte de sua história, que, na maioria dos casos, vem acompanhada de outras formas de violência extra e intrafamiliar (Saywitz \& cols., 2000). Dessa forma, a melhoria das crianças não depende apenas da eficácia/efetividade do tratamento, mas do funcionamento dos adultos cuidadores da criança. A saúde mental dos pais, conflito conjugal, dinâmica familiar, presença de eventos estressores, o nível socioeconômico da família, fatores culturais e comunitários influenciam no grau e manutenção da melhoria. No Brasil, estudos sobre avaliação de métodos terapêuticos são escassos, sendo que, além das dificuldades anteriormente citadas, verificase a ausência de instrumentos psicológicos validados e eficazes para avaliação clínica das vítimas (Habigzang, Hatzenberger, 
Dala Corte, Stroeher \& Koller, 2006; Habigzang, Stroeher, Dala Corte, Hatzenberger \& Koller, no prelo).

Apesar dos obstáculos descritos, as pesquisas que utilizam a Terapia Cognitivo-Comportamental (TCC) como forma de tratamento têm apresentado melhores resultados quando comparada com outras formas de tratamento não-focais para crianças e adolescentes com sintomas de ansiedade, depressão e problemas comportamentais decorrentes de violência sexual (Cohen, Mannarino \& Knudsen, 2005; Deblinger, Stauffer \& Steer, 2001; Saywitz \& cols., 2000). Além disso, TCC focada no trauma tem apresentado alta eficácia na redução de sintomas do transtorno do estresse pós-traumático (TEPT) (Cohen, 2003; Cohen, Mannarino \& Rogal, 2001) e na reestruturação de crenças disfuncionais com relação à experiência abusiva (Celano, Hazzard, Campbell \& Lang, 2002).

Para avaliar uma intervenção com 49 crianças abusadas sexualmente, Cohen e Mannarino (2000) testaram vítimas com idade entre 7 e 14 anos e seus cuidadores primários não-abusivos. Durante o período de 12 semanas, a amostra foi dividida em duas formas de tratamento: TCC focada no abuso e terapia de apoio não-focal. As condições de tratamento foram fixadas aleatoriamente e monitoradas por terapeutas intensivamente treinados e supervisionados. As crianças foram avaliadas antes e depois do tratamento por meio de uma variedade de instrumentos. Entre os resultados de Cohen e Mannarino, foi constatado que o desenvolvimento de sintomas e a resposta ao tratamento são influenciados pelo sofrimento emocional dos pais com relação ao abuso, o apoio dos pais à criança e as crenças que a criança apresenta com relação ao abuso. Com relação às formas de tratamento, a TCC obteve resultados superiores quando comparada à outra forma de intervenção quanto à redução de sintomas de depressão e ansiedade, uma vez que a TCC teve como foco da intervenção a reestruturação de atribuições e percepções distorcidas sobre abuso sexual.

Em um estudo adicional, seguindo a mesma metodologia, a durabilidade dos efeitos da TCC foi avaliada após um ano do término do tratamento (Cohen \& cols., 2005). Participaram do estudo 82 crianças abusadas sexualmente, com idades entre 8 e 15 anos, alocadas aleatoriamente em duas formas de tratamento: TCC focada no trauma e terapia de apoio não-focal, ambas com duração de 12 semanas. A sintomatologia das crianças foi reavaliada após seis e 12 meses do término do tratamento e os resultados apontaram que o grupo em tratamento com TCC apresentou significativa redução de sintomas de depressão, ansiedade e problemas sexuais após as 12 sessões, quando comparado ao grupo em terapia de apoio não-focal. Após os seis meses do término do tratamento, foi constatada uma melhora significativamente maior nos sintomas de ansiedade, depressão, problemas sexuais e dissociação e após 12 meses houve melhoras significativas nos sintomas de TEPT e dissociação. Esses resultados apontam a durabilidade da efetividade da TCC.

A investigação das relações entre a exposição à terapia focada no abuso e as mudanças na sintomatologia em crianças abusadas sexualmente foi realizada também por Lanktree e Briere (1995). Os objetivos da pesquisa eram verificar se a sintomatologia depressão, ansiedade e transtorno do estresse pós-traumático diminuíam com o tempo de tratamento e se essa mudança era de fato efeito da terapia ou representava simplesmente efeitos da passagem do tempo. Os participantes desse estudo foram crianças com idade entre 8 e 15 anos. Participaram da pesquisa 105 crianças. Destas, $84,8 \%$ eram meninas com idade média de 11,6 anos. Entre os casos incluídos na amostra, 46,7\% eram incestuosos e 44,8\% dos casos envolviam sexo oral, anal ou penetração vaginal. As crianças preencheram o Children's Depression Inventory (CDI - Inventário de Depressão Infantil) e o Trauma Symptom Checklist for Children (TSCC-Checklist de sintomas de trauma para crianças) em intervalos de três meses. Os participantes receberam tratamento individual focado no abuso e, em muitos casos, terapia familiar e de grupo.

O estudo de Lanktree e Briere (1995) demonstrou que os sintomas psicológicos decorrentes do abuso sexual diminuíram durante o processo de terapia focado no abuso. Essa redução do efeito do trauma variou de acordo com o tipo de sintoma e tempo de terapia. Os participantes que apresentavam sintomas de ansiedade e TEPT responderam mais rapidamente e de forma constante ao tratamento, enquanto que os que apresentavam questões relacionadas à sexualidade levaram um período de tempo maior. Outro achado interessante refere-se ao intervalo de tempo. Quanto menor o tempo entre o fim do abuso e começo da terapia, melhores foram os resultados no pré-teste nas escalas de depressão, raiva e dissociação, ou seja, crianças que permanecem mais tempo sem uma intervenção tendem a dissociar mais e apresentar maior sintomatologia de depressão e sentimentos de raiva. Esses achados sugerem que o trauma gerado pelo abuso pode produzir um crescimento dos efeitos negativos na ausência de um tratamento apropriado. Tal fato parece indicar que a mera passagem do tempo, sem tratamento, não reduz a intensidade dos sintomas na escala de tempo investigada (Lanktree \& Briere, 1995).

Alguns estudos apontam que a TCC no formato grupal tem obtido resultados positivos no tratamento de crianças e adolescentes vítimas de violência sexual. $\mathrm{O}$ grupo oportuniza reduzir o sentimento de diferença em relação aos pares, bem como oferece espaços para que as vítimas possam relatar sentimentos referentes ao abuso; discutir crenças quanto à culpa pela experiência abusiva. Além disso, possibilita integrar e reestruturar a memória traumática, desenvolver habilidades preventivas a outras situações abusivas; e elaborar sentimentos de isolamento e estigmatização (Habigzang \& Caminha, 2004; Kruczek \& Vitanza, 1999; McCrone \& cols., 2005; McGain \& McKinzey, 1995; Smith, 1993/1996).

Apesar das evidências apontadas pelos estudos internacionais de que a terapia cognitivo-comportamental é efetiva para o tratamento de crianças e adolescentes vítimas de abuso sexual, não se encontram estudos empíricos de avaliação de modelos de intervenção criados e aplicados no Brasil para essa população. O presente trabalho tem como objetivo avaliar o efeito de um modelo de grupoterapia cognitivo-comportamental para meninas vítimas de abuso sexual intrafamiliar.

\section{Método}

\section{Delineamento}

A avaliação do efeito da intervenção nos sintomas de depressão, ansiedade, transtorno do estresse pós-traumático, bem como nas crenças em relação ao abuso, nas participantes deste estudo, foi realizada pela aplicação dos instrumentos psicológicos antes do início da intervenção e após cada etapa da grupoterapia. Foram aplicadas medidas repetidas em quatro tempos distintos durante o processo: pré-teste (avaliação 
inicial), pós-teste 1 (após psicoeducação - sete semanas), pósteste 2 (após treino de inoculação do estresse - 12 semanas ) e pós-teste 3 (após prevenção à recaída - 20 semanas).

\section{Participantes}

O estudo foi realizado com 10 meninas, com idade entre 9 e 13 anos, que foram submetidas a pelo menos um episódio de abuso sexual intrafamiliar. Os critérios de inclusão para o estudo foram: presença de pelo menos um episódio de abuso sexual intrafamiliar, sexo feminino e idade entre 9 e 16 anos. A seleção das participantes ocorreu por meio do contato com o Programa Sentinela e o Conselho Tutelar de uma cidade do Estado do Rio Grande do Sul.

O Programa Sentinela encaminhou oito casos para a pesquisa. Desses, apenas dois foram incluídos no estudo, uma vez que três casos configuravam-se como abuso sexual extrafamiliar e o restante não residia mais no endereço informado e não foi possível localizar. Os casos que não foram incluídos no estudo foram reencaminhados para o Programa Sentinela. O Conselho Tutelar permitiu que a equipe de pesquisa realizasse um levantamento em seu arquivo para selecionar participantes. Foram selecionados casos denunciados entre 2000 e 2004, totalizando 17. O contato foi estabelecido por meio de correspondência, na qual foi oferecido atendimento psicológico gratuito. Apenas uma menina compareceu ao atendimento agendado. Cinco correspondências foram devolvidas, pois a família não residia mais no endereço informado. A equipe enviou um segundo convite para atendimento por meio do Conselho Tutelar para as outras 11 meninas, mas elas não compareceram. Os conselheiros tutelares encaminharam sete casos de abuso sexual que ainda estavam sendo acompanhados e estes foram incluídos na amostra.

A idade do início do abuso sexual variou entre 5 e 11 anos, sendo que, das 10 participantes, cinco meninas estavam entre 7 e 8 anos. O perpetrador da violência foi: pai biológico (dois casos), tio (três casos), avô (três casos), irmão (um caso) e casal de padrinhos (um caso). Dos 10 casos, nove participantes foram vítimas de mais de um episódio de abuso sexual e a violência teve duração de pelo menos um ano. O tipo de abuso sexual variou entre os casos, sendo que em sete ocorreram toques, manipulação de genitais e assédio e em três, relações sexuais com penetração. Uma das participantes foi vítima de pornografia infantil, sendo fotografada pelo agressor. Também foi verificado que oito meninas foram vítimas de abusos psicológicos e físicos e todas sofreram ameaças para manter o abuso sexual em sigilo. Após a denúncia do abuso sexual, seis meninas foram afastadas do convívio com os pais, sendo que cinco meninas foram abrigadas e uma passou a residir com a tia. As outras quatro participantes permaneceram com os pais, que se organizaram de forma protetiva, afastando as meninas dos agressores.

\section{Instrumentos}

Os instrumentos utilizados para avaliação do efeito da intervenção foram:

1) Entrevista semi-estruturada inicial constituída por duas partes: na primeira parte, o objetivo é estabelecer um vínculo terapêutico com a participante, criando um espaço seguro baseado em uma relação de confiança. A segunda parte está baseada na entrevista publicada pelo The Metropolitan Toronto Special Committee on Child Abuse (1995), traduzida para o português e adaptada por Kristensen (1996). Essa entrevista tem como principal objetivo obter o relato da participante com relação ao abuso sexual, bem como mapear a frequiência e a dinâmica dos episódios abusivos.

2) Children's Attributions and Perceptions Scale (CAPS - Escala de atribuições e percepções de crianças): foi desenvolvido para mensurar questões específicas do abuso em crianças que sofreram abuso sexual (Mannarino, Cohen \& Berman, 1994). O instrumento é uma entrevista semi-estruturada constituída por 18 itens, sendo que a criança responde cada item em uma escala de cinco respostas [escala Likert, que varia entre nunca (0) e sempre (4)]. Quatro aspectos são avaliados em quatro subescalas: sentimentos de diferença com relação aos pares; confiança nas pessoas; auto-atribuição dos eventos negativos (auto-culpabilização pelo abuso); e percepção de credibilidade dos outros em si. Os itens da entrevista foram traduzidos para o português por um pesquisador bilíngüe e depois traduzidos de volta para o inglês por outro. As versões foram comparadas, ajustadas e aplicadas em cinco meninas com idade entre 10 e 13 anos para verificar a compreensão do instrumento.

3) Inventário de Depressão Infantil (CDI): foi elaborado por Kovacs (1992), adaptado do Beck Depression Inventory para adultos. O objetivo do CDI é detectar a presença e a gravidade do transtorno depressivo. Destina-se a identificar alterações afetivas em crianças e adolescentes, dos 7 aos 17 anos de idade. Esse inventário é composto por 27 itens, cada um com três opções de resposta, pontuadas de 0 a 2. A consistência interna descrita por Kovacs mostrou-se adequada $(\alpha=0,86)$, e o ponto de corte do CDI foi estabelecido em 19 pontos. O CDI já foi adaptado, para uso no Brasil, por Gouveia, Barbosa, Almeida e Gaião (1995) e vem demonstrando características psicométricas adequadas. Esse instrumento também foi utilizado em pesquisas no Rio Grande do Sul (Giacomoni, 1998), apresentando um alpha de Cronbach $=0,82$ e uma correlação negativa com nível de satisfação de vida $(r=-0,25)$.

4) Escala de Estresse Infantil (ESI): A escala é composta por 35 itens relacionados às seguintes reações do estresse: físicas, psicológicas, psicológicas com componente depressivo e psicofisiológica, em crianças entre 6 e 14 anos. A resposta ao item é feita por meio de uma escala Likert de cinco pontos, na qual a criança pinta um círculo dividido em quatro partes, conforme a frequiência com que os participantes experimentam os sintomas apontados pelos itens (Lipp \& Lucarelli, 1998).

5) Inventário de Ansiedade Traço-Estado para crianças (IDATE-C): O inventário é constituído de duas escalas do tipo auto-avaliação, que visam a medir dois conceitos distintos de ansiedade: traço e estado (foi elaborado por Spielberger em 1970, e adaptado para uso no Brasil por Biaggio \& Spielberger, em 1983). A escala de ansiedade-estado indica como a criança se sente em um determinado momento do tempo, medindo estados transitórios de sentimentos subjetivos, enquanto a escala de ansiedade-traço avalia como a criança geralmente se sente, medindo diferenças relativamente estáveis em susceptibilidade à ansiedade. Cada escala é composta por 20 itens e cada item é constituído por três afirmações que representam diferentes intensidades do sintoma. No presente 


\section{F. Habigzang e cols}

Tabela 1. Descrição das sessões da grupoterapia.

\begin{tabular}{|c|c|c|}
\hline Etapa & Sessão & Atividades \\
\hline \multirow{7}{*}{$\begin{array}{l}\mathbf{P} \\
\mathbf{S} \\
\mathbf{i} \\
\mathbf{c} \\
\mathbf{o} \\
\mathbf{e} \\
\mathbf{d} \\
\mathbf{u} \\
\mathbf{c} \\
\mathbf{a} \\
\mathbf{c} \\
\tilde{\mathbf{a}} \\
\mathbf{0}\end{array}$} & $1^{\mathrm{a}}$ & $\begin{array}{l}\text { Qualquer dinâmica de grupo que favoreça a apresentação das participantes; } \\
\text { Estabelecimento do contrato } \\
\text { Discussão sobre a identidade do grupo; } \\
\text { Mapeamento das expectativas das participantes. Dispositivo: construção de um cartaz; } \\
\text { Discussão e estabelecimento dos objetivos do grupo. }\end{array}$ \\
\hline & $2^{\mathrm{a}}$ & $\begin{array}{l}\text { Dinâmica de grupo para desenvolver a confiança. Dispositivo: técnica da caminhada em duplas com olhos vendados (Smith, 1993/ 1996); } \\
\text { Relato das situações abusivas de cada participante; } \\
\text { Isenção da culpa; } \\
\text { Abordar terapeuticamente o impacto afetivo da revelação no grupo }\end{array}$ \\
\hline & $3^{\mathrm{a}}$ & $\begin{array}{l}\text { Abordar as reações da família e demais pessoas significativas depois da revelação e construir um mapeamento das possíveis mudanças } \\
\text { na configuração familiar; } \\
\text { Reestruturação cognitiva de crenças relacionadas à culpa e vergonha (construção de cartões com explicações alternativas para o abuso } \\
\text { que desculpabilize as participantes) }\end{array}$ \\
\hline & $4^{\mathrm{a}}$ & $\begin{array}{l}\text { Psicoeducação quanto ao modelo cognitivo-comportamental - abordagem de estados emocionais. Dispositivo: jogo das emoções, no qual } \\
\text { as participantes relacionam situações e emoções correspondentes, contribuindo para que estas aprendam a nomear seus sentimentos; } \\
\text { Abordagem terapêutica dos sentimentos com relação ao abusador. Dispositivo: construção do abusador com massa de modelar e role-play entre } \\
\text { cada participante com o boneco, no qual cada uma possa expressar seus sentimentos em um ambiente seguro (Knell \& Ruma, 1996/1999); } \\
\text { Entrega do automonitoramento, no qual será solicitado o registro de situações-problema e sentimentos identificados }\end{array}$ \\
\hline & $5^{\mathrm{a}}$ & $\begin{array}{l}\text { Discussão dos registros de automonitoramentos; } \\
\text { Psicoeducação quanto ao modelo cognitivo-comportamental - abordagem dos pensamentos e sua relação com sentimentos; } \\
\text { Identificação de pensamentos com relação ao abuso (tríade cognitiva) e reestruturação cognitiva de pensamentos disfuncionais; } \\
\text { Entrega do automonitoramento, no qual será registrado: situações-problema, pensamentos e sentimentos relacionados }\end{array}$ \\
\hline & $6^{\mathrm{a}}$ & $\begin{array}{l}\text { Discussão dos registros de automonitoramentos; } \\
\text { Psicoeducação quanto ao modelo cognitivo-comportamental - abordagem dos comportamentos e sua relação com pensamentos e sentimentos; } \\
\text { Mapeamento escrito das principais mudanças de comportamentos, pensamentos e sentimentos decorrentes das experiências abusivas; } \\
\text { Psicoeducação quanto ao problema (estabelecimento de relações entre abuso/trauma com as mudanças identificadas no mapeamento) }\end{array}$ \\
\hline & $7 \underline{a}$ & $\begin{array}{l}\text { Psicoeducação quanto ao modelo cognitivo-comportamental - abordagem de reações físicas que podem estar associadas a pensamentos, } \\
\text { sentimentos e comportamentos; } \\
\text { Aprendizagem de técnicas de relaxamento muscular e de respiração para controle da ansiedade (Vera \& Vila, 1996); } \\
\text { Discussão dos automonitoramentos e construção de histórias em quadrinhos a partir de situações registradas, nas quais apareçam os senti- } \\
\text { mentos, pensamentos, comportamentos e reações físicas para integrar o modelo cognitivo-comportamental (Knell \& Ruma, 1996/1999); } \\
\text { Entrega do automonitoramento }\end{array}$ \\
\hline $\begin{array}{l}\mathbf{I} \\
\mathbf{n} \\
\mathbf{o} \\
\mathbf{c} \\
\mathbf{u} \\
\mathbf{l}\end{array}$ & $8^{\mathrm{a}}$ & $\begin{array}{l}\text { Treino de inoculação de estresse (o TIE é utilizado como dispositivo para ativar a memória traumática e detalhar os estímulos desencadeantes } \\
\text { de lembranças intrusivas, possibilitando às participantes uma sensação de controle da intensidade das emoçôes associadas). Nesta técnica } \\
\text { cada participante apresentam, de forma gradual, as situações abusivas experienciadas através do relato oral ou por escrito; } \\
\text { Reestruturação cognitiva das memórias traumáticas, na qual as participantes possam elaborar explicações funcionais para o evento } \\
\text { traumático e romper o ciclo entre estímulos relacionados ao abuso e a re-experienciação deste }\end{array}$ \\
\hline $\begin{array}{l}\mathbf{a} \\
\mathbf{c} \\
\tilde{\mathbf{a}} \\
\mathbf{0}\end{array}$ & $9^{\mathrm{a}}$ & $\begin{array}{l}\text { Treino de inoculação de estresse } \\
\text { Reestruturação cognitivo-comportamental (construção do "botão de emergência" com estratégias cognitivas e comportamentais para } \\
\text { lidar com lembranças intrusivas do abuso). }\end{array}$ \\
\hline $\mathbf{S}$ & $10^{\mathrm{a}}$ & Treino de inoculação de estresse \\
\hline $\begin{array}{l}\mathbf{t} \\
\mathbf{r}\end{array}$ & $11^{\mathrm{a}}$ & Reestruturação cognitivo-comportamental \\
\hline $\begin{array}{l}\text { e } \\
\mathbf{S} \\
\mathbf{S}\end{array}$ & $12^{\mathrm{a}}$ & $\begin{array}{l}\text { Treino de inoculação de estresse (relato do pior momento); } \\
\text { Reestruturação cognitivo-comportamental (aprendizagem da técnica cognitiva das "caixas de memórias positivas e negativas" que } \\
\text { existem em nossas cabeças e que podem ser contrapostas) }\end{array}$ \\
\hline $\mathbf{P}$ & 13 a & Oficina educação sexual, na qual são abordadas questões referentes ao autocuidado, mudanças físicas da puberdade e métodos contraceptivos \\
\hline $\begin{array}{l}\mathbf{r} \\
\mathbf{e} \\
\mathbf{v}\end{array}$ & $14 \mathrm{a}$ & $\begin{array}{l}\text { Treino de habilidades sociais focadas em medidas protetivas (identificação de situações de risco e ensaio cognitivo e comportamental } \\
\text { de estratégias de proteção contra futuras revitimizações) }\end{array}$ \\
\hline $\begin{array}{l}\mathbf{e} \\
\mathbf{n} \\
\mathbf{c} \\
\tilde{\mathbf{a}}\end{array}$ & $15^{\mathrm{a}}$ & $\begin{array}{l}\text { Oficina sobre o Estatuto da criança e do adolescente (discussão dos direitos fundamentais e leis previstas em casos de abuso sexual); } \\
\text { Dramatização de audiência (abordar a possibilidade de participação de audiências, esclarecendo dúvidas e preparando as participantes } \\
\text { para tal situação) }\end{array}$ \\
\hline $\mathbf{0}$ & $16^{\mathrm{a}}$ & Oficina de psicomotricidade (será convidado um profissional da educação física para conduzir a atividade) \\
\hline $\begin{array}{l}\text { à } \\
\mathbf{R} \\
\text { e }\end{array}$ & $17^{\mathrm{a}}$ & $\begin{array}{l}\text { Feedback da oficina de psicomotricidade; } \\
\text { Prevenção à recaída (retomada as estratégias cognitivas e comportamentais aprendidas no contexto grupal através da dramatização de } \\
\text { situações-problema) }\end{array}$ \\
\hline c & $18^{\mathrm{a}}$ & Prevenção à recaída e escolha de um adulto-referência para solicitar ajuda em situações de risco \\
\hline a & $19 \underline{\underline{a}}$ & Abordar as perspectivas com relação ao futuro das participantes e reestruturar possíveis crenças distorcidas. \\
\hline $\begin{array}{l}\text { d } \\
\mathbf{a}\end{array}$ & $20^{\mathrm{a}}$ & $\begin{array}{l}\text { Auto-avaliação através de registro escrito das mudanças percebidas antes e depois da grupoterapia em relação a si, como se relaciona } \\
\text { com os outros e visão do futuro. }\end{array}$ \\
\hline
\end{tabular}


estudo, apenas a escala de ansiedade-estado foi aplicada e analisada, uma vez que o objetivo da intervenção foi reduzir sintomas relacionados ao estado de ansiedade.

6) Entrevista estruturada com base no DSM IV/SCID para avaliação de transtorno do estresse pós-traumático: Os critérios diagnósticos estabelecidos pelo Manual Diagnóstico e Estatístico dos Transtornos Mentais (DSM-IV, 2002) são utilizados como base para identificação da presença dos sintomas que compõem o transtorno. A tradução para o português dessa entrevista foi desenvolvida por Del Ben e cols. (2001). A equipe de pesquisa fez algumas alterações na linguagem utilizada na entrevista para facilitar a compreensão das perguntas pelas crianças e adolescentes, tendo como base os resultados do estudo piloto, no qual a entrevista foi aplicada em cinco meninas com idade entre 10 e 13 anos.

\section{Procedimentos}

O projeto desta pesquisa foi avaliado e aprovado pelo Comitê de Ética da Universidade Federal do Rio Grande do Sul. Após a aprovação, foi realizado o treinamento teóricometodológico e ético da equipe que auxiliou na pesquisa. Em seguida, o contato com o Conselho Tutelar e Programa Sentinela foi estabelecido para os encaminhamentos.

Após a seleção das participantes, foi realizada a avaliação clínica individual pelas assistentes de pesquisa (Habigzang \& cols., no prelo). A avaliação foi composta por três encontros com duração de uma hora cada e com frequiência semanal. Os encontros foram supervisionados em reuniões semanais com a equipe, nas quais os casos e procedimentos eram discutidos. A ordem de aplicação dos instrumentos foi alterada aleatoriamente na segunda e terceira entrevistas para evitar o efeito de ordem nos resultados. Em cada encontro, foram aplicados os seguintes instrumentos: 10 encontro, entrevista semi-estruturada inicial, que foi gravada e transcrita; 2o encontro, inventários de depressão (CDI), de ansiedade (IDATE-C) e entrevista semi-estruturada (CAPS); 3o encontro, entrevista estruturada com base no DSM-IV para avaliação do transtorno do estresse pós-traumático e escala de estresse infantil.

Após a avaliação clínica individual, as participantes foram encaminhadas para a intervenção grupoterápica. Foram formados dois grupos de acordo com o turno em que as meninas freqüentavam a escola. A grupoterapia é um modelo de intervenção grupal cognitivo-comportamental testado experimentalmente, foi uma adaptação do modelo proposto por Habigzang e Caminha (2004). Foi composto por 20 sessões com frequiência semanal. As sessões tiveram atividades semi-estruturadas, com duração de uma hora e 30 minutos. Os objetivos da intervenção foram: reestruturar pensamentos, emoções e comportamentos disfuncionais relacionados à experiência de violência sexual; reduzir sintomas de transtorno do estresse pós-traumático, estresse, depressão e ansiedade; e aprender habilidades de autoproteção para prevenir futuras revitimizações. O processo grupoterápico foi dividido em três etapas conforme as técnicas empregadas: Etapa 1 - Psicoeducação (sete sessões); Etapa 2 - Treino de inoculação do estresse (cinco sessões); e Etapa 3 - Prevenção à recaída (oito sessões). A descrição de cada sessão está apresentada na Tabela 1. Além do processo terapêutico desenvolvido com as meninas, ocorreram, paralelamente, quatro encontros, com freqüência mensal, de psicoeducação para os cuidadores não-abusivos das participantes. O objetivo desses encontros foi compartilhar com os cuidadores informações relevantes sobre o abuso sexual e consequiências para o desenvolvimento das crianças, bem como construir estratégias de proteção e fortalecer o apoio afetivo destes com as meninas (Habigzang, \& cols., 2006).

As participantes foram reavaliadas clinicamente ao término de cada etapa da grupoterapia. A reavaliação clínica ocorreu em um encontro individual com a mesma assistente de pesquisa que realizou a avaliação inicial em horário diferente do grupo. Nessa avaliação, foram aplicados os mesmos instrumentos da avaliação inicial, com ordem aleatória, para verificar sintomas de depressão, ansiedade, transtorno do estresse pós-traumático e crenças disfuncionais com relação ao abuso.

\section{Resultados e Discussão}

Os dados obtidos por meio dos instrumentos CDI, IDATE-C, ESI, CAPS e entrevista para TEPT foram inicialmente submetidos a análises descritivas, nas quais foram calculados a média, o desvio padrão e a mediana de cada instrumento em cada tempo. Após as análises descritivas, os dados foram submetidos ao Teste de Friedman e os resultados tendencialmente significativos $(p<0,10)$ ou significativos $(p<0,05)$ foram analisados por meio do teste estatístico não-paramétrico Wilcoxon, utilizado para amostras pareadas. A combinação de todas as medidas de cada instrumento foi analisada (pré e pós 1 ; pré e pós 2 , pré e pós 3 , pós 1 e pós 2 , pós 1 e pós 3 e pós 2 e pós 3 ) e os resultados tendencialmente significativos ou significativos são apresentados e discutidos a seguir.

Os sintomas de depressão, avaliados por meio do Inventário de Depressão Infantil (CDI), apresentaram redução tendencialmente significativa entre pré-teste e o pós-teste 3 $(Z=1,79, p<0,10)$ (veja Tabela 2). Entretanto, foi encontrada diferença significativa nos resultados entre o pós-teste 1 e o pós-teste $3(Z=2,20, p<0,05)$. Esse resultado sugere que, após as sete sessões de psicoeducação (pós 1), iniciou-se a redução de sintomas de depressão.

Tabela 2. Média, desvio padrão e mediana do Inventário de Depressão (CDI) e de Ansiedade (IDATE-C-Estado).

\begin{tabular}{|c|c|c|c|c|c|c|c|c|c|c|c|c|}
\hline \multirow{2}{*}{ Instrumento } & \multicolumn{3}{|c|}{ Pré-teste } & \multicolumn{3}{|c|}{ Pós-teste 1} & \multicolumn{3}{|c|}{ Pós-teste 2} & \multicolumn{3}{|c|}{ Pós-teste 3} \\
\hline & $x$ & $d p$ & $M d$ & $x$ & $d p$ & $M d$ & $x$ & $d p$ & $M d$ & $x$ & $d p$ & $M d$ \\
\hline CDI & 18,50 & 12,22 & 15,00 & 12,60 & 7,15 & 14,00 & 9,90 & 7,22 & 9,50 & 8,50 & 5,82 & 8,00 \\
\hline IDATE-C Estado & 33,80 & 7,21 & 35,50 & 35,50 & 7,41 & 35,00 & 31,40 & 7,01 & 30,50 & 30,00 & 5,58 & 31,50 \\
\hline
\end{tabular}


A análise dos sintomas de ansiedade, avaliados pelo Inventário de Ansiedade Infantil/Estado (IDATE), também apresentou diferença significativa entre pós-teste 1 e pósteste $3(Z=2,08, p<0,05)$. Dessa forma, houve uma redução significativa de sintomas de ansiedade a partir da etapa da psicoeducação (pós 1). A Tabela 2 apresenta os resultados da análise descritiva do IDATE-C.

A redução significativa de sintomas de depressão e ansiedade-estado entre a etapa de psicoeducação e o final da intervenção aponta que a reestruturação de crenças disfuncionais relacionadas com culpa e diferença em relação aos pares enfatizada nas primeiras sessões, bem como a compreensão do que é abuso sexual e a exploração de sentimentos e percepções sobre essa experiência, tiveram impacto positivo nesses sintomas. Tal resultado está em consonância com os estudos que apontam que as percepções distorcidas da criança, em relação ao abuso, estão relacionadas com maior sintomatologia de depressão e ansiedade (Cohen \& Mannarino, 2000; Heflin \& Deblinger, 1996/1999; Runyon \& Kenny, 2002; Valle \& Silovsky, 2002).

Os sintomas de estresse infantil, avaliados pela Escala de Stress Infantil (ESI), apresentaram redução significativa entre o pré-teste e pós-teste $3(Z=2,19, p<0,05)$. A Tabela 3 apresenta os resultados das análises descritivas de cada subescala e total do ESI. Esse resultado pode sugerir que apenas o encaminhamento para atendimento psicológico pode reduzir o estresse da criança, pois pode representar uma oportunidade de sentir-se melhor e despertar a esperança de superar o evento. Contudo, a análise de cada subescala do instrumento apontou diferença significativa apenas naquelas que avaliam sintomas psicológicos $(Z=2,39, p<0,05)$ e psicofisiológicos $(Z=2,30, p<0,05)$. Não foi encontrada diferença significativa em sintomas físicos e psicológicos com componente depressivo de estresse. Esse dado pode sugerir que as técnicas empregadas ao longo do processo terapêutico promovem melhoras em sintomas psicológicos e psicofisiológicos relacionados ao stress.

Os sintomas de transtorno do estresse pós-traumático apresentaram redução significativa nas três categorias que o constituem: revivência do trauma, evitação e excitação aumentada. Os sintomas da categoria "revivência do evento traumático" apresentaram uma redução tendencialmente significativa entre o pós-teste 2 e $3(Z=1,85, p<0,10)$ e significativa entre o pós-teste 1 e $3(Z=2,46, p<0,05)$. Esse dado sugere que o treino de inoculação do estresse é relevante para a redução dos sintomas de reexperienciação do trauma. Os sintomas da categoria "evitação" apresentaram redução significativa entre o pré-teste e pós-teste $3(Z=2,41, p<0,05)$. Essa redução a partir do pré-teste aponta a importância de entrar em contato com as lembranças traumáticas em um contexto seguro, por meio do relato verbal ou escrito, para o controle das reações emocionais. As participantes relataram o abuso sexual na entrevista inicial e detalharam tal relato ao longo do processo, reduzindo a percepção sobre a evitação de pessoas, locais e lembranças da experiência traumática. Os sintomas da categoria "excitação aumentada" apresentaram redução significativa entre o pós-teste 2 e $3(Z=1,98, p<0,05)$ e entre o pós-teste 1 e $3(Z=2,16, p<0,05)$.

A Tabela 4 apresenta a análise descritiva de cada categoria de sintomas do TEPT. Contudo, ao avaliar os resultados, considerando presença ou ausência do quadro de estresse pós-traumático, por meio do Teste McNemar para escala binária, verificou-se uma redução tendencialmente significativa entre o pré e pós-teste $3(p<0,10)$, uma vez que no pré-teste foi verificado que sete das 10 participantes apresentavam quadro de TEPT e, ao término do processo terapêutico, o número de crianças com TEPT havia sido reduzido para dois. A redução dos sintomas de TEPT encontrada neste estudo está em consonância com os estudos que apontam a eficácia da TCC na melhora desse transtorno (Astin \& Resick, 2002; Cohen, 2003; Cohen \& cols., 2001).

Por fim, verificou-se diferença significativa nas crenças das participantes sobre a experiência abusiva, avaliadas por meio do CAPS. Houve uma redução significativa na percepção das meninas sobre a culpa entre o pós 1 e pós-teste $3(Z=2,11$, $p<0,05)$ e tendencialmente significativa na percepção de diferença em relação aos pares nesse mesmo intervalo de tempo $(Z=1,88, p<0,10)$. A percepção das participantes sobre a credibilidade dos outros em si aumentou significativamente entre o pré e pós-teste $3(Z=2,25, p<0,05)$. Também houve um aumento significativo na percepção de confiança das meninas em outras pessoas entre o pré e pós-teste $3(Z=2,71, p<0,05)$.

Tabela 3. Média, desvio padrão e mediana da Escala de Stress Infantil (ESI).

\begin{tabular}{|c|c|c|c|c|c|c|c|c|c|c|c|c|}
\hline \multirow{2}{*}{ ESI } & \multicolumn{2}{|c|}{ Pré-teste } & \multicolumn{4}{|c|}{ Pós-teste 1} & \multicolumn{2}{|c|}{ Pós-teste 2} & \multicolumn{4}{|c|}{ Pós-teste 3} \\
\hline & $x$ & $d p$ & Md & $x$ & $d p$ & Md & $x$ & $d p$ & $M d$ & $x$ & $d p$ & $M d$ \\
\hline R. Físicas & 8,60 & 3,06 & 8,50 & 8,10 & 4,04 & 7,50 & 8,30 & 5,64 & 10,00 & 6,50 & 5,52 & 7,00 \\
\hline R. psicológicas & 16,90 & 4,23 & 18,00 & 17,30 & 5,23 & 18,00 & 11,60 & 6,33 & 12,00 & 8,20 & 6,43 & 8,00 \\
\hline $\begin{array}{l}\text { R. psicológicas c/ comp. } \\
\text { Depressivo }\end{array}$ & 9,20 & 4,80 & 8,00 & 10,20 & 6,21 & 8,00 & 9,30 & 5,60 & 7,50 & 7,90 & 6,76 & 7,00 \\
\hline R. psicofisiológicas & 10,60 & 3,41 & 10,00 & 12,90 & 6,69 & 14,00 & 10,30 & 5,46 & 9,00 & 7,60 & 6,64 & 7,50 \\
\hline Total & 45,30 & 9,89 & 45,50 & 48,30 & 17,57 & 47,50 & 39,50 & 20,45 & 43,50 & 30,20 & 23,31 & 28,00 \\
\hline
\end{tabular}

Tabela 4. Média, desvio padrão e mediana das categorias de sintomas de Transtorno do Estresse Pós-Traumático (TEPT).

\begin{tabular}{|c|c|c|c|c|c|c|c|c|c|c|c|c|}
\hline \multirow{2}{*}{ TEPT } & \multicolumn{3}{|c|}{ Pré-teste } & \multicolumn{3}{|c|}{ Pós-teste 1} & \multicolumn{3}{|c|}{ Pós-teste 2} & \multicolumn{3}{|c|}{ Pós-teste 3} \\
\hline & $x$ & $d p$ & $M d$ & $x$ & $d p$ & $M d$ & $x$ & $d p$ & $M d$ & $x$ & $d p$ & $M d$ \\
\hline Revivência & 2,90 & 1,10 & 2,50 & 3,40 & 1,35 & 3,00 & 2,70 & 1,95 & 3,00 & 1,70 & 1,34 & 1,50 \\
\hline Evitação & 3,60 & 1,65 & 3,50 & 3,60 & 1,43 & 3,50 & 3,70 & 1,49 & 4,00 & 2,20 & 1,62 & 2,00 \\
\hline Hipevigilância & 3,60 & 1,43 & 4,00 & 3,70 & 1,34 & 4,00 & 3,50 & 1,08 & 4,00 & 2,60 & 1,35 & 3,00 \\
\hline
\end{tabular}


Intervenção Psicológica em Abuso Sexual

Tabela 5. Média, desvio padrão e mediana das percepções das participantes relacionadas ao abuso avaliadas pela entrevista CAPS.

\begin{tabular}{|c|c|c|c|c|c|c|c|c|c|c|c|c|}
\hline \multirow{2}{*}{ CAPS } & \multicolumn{3}{|c|}{ Pré-teste } & \multicolumn{3}{|c|}{ Pós-teste 1} & \multicolumn{3}{|c|}{ Pós-teste 2} & \multicolumn{3}{|c|}{ Pós-teste 3} \\
\hline & $x$ & $d p$ & $M d$ & $x$ & $d p$ & $M d$ & $X$ & $d p$ & $M d$ & $x$ & $d p$ & $M d$ \\
\hline Dif/ pares & 7,90 & 1,73 & 8,00 & 8,50 & 3,24 & 8,00 & 6,70 & 2,36 & 7,50 & 7,10 & 2,77 & 6,50 \\
\hline Sent. culpa & 7,90 & 2,28 & 8,50 & 8,60 & 3,10 & 7,50 & 6,70 & 2,71 & 6,50 & 6,80 & 2,62 & 6,50 \\
\hline Credibilidade & 11,60 & 3,17 & 11,50 & 9,00 & 2,94 & 8,00 & 10,60 & 3,17 & 10,50 & 8,40 & 3,47 & 7,00 \\
\hline Confiança & 13,10 & 3,28 & 13,00 & 10,50 & 4,43 & 11,50 & 10,50 & 3,69 & 11,50 & 9,30 & 2,83 & 8,50 \\
\hline
\end{tabular}

Esses resultados sugerem a importância da reestruturação cognitiva, bem como o vínculo seguro com os terapeutas e com o grupo de pares na modificação de pensamentos e percepções disfuncionais. A Tabela 5 apresenta os resultados da análise descritiva do CAPS.

A avaliação do efeito da intervenção obtida nos resultados sobre depressão, ansiedade, estresse, transtorno do estresse pós-traumático e crenças disfuncionais, refletiram as mudanças qualitativas positivas observadas ao longo do processo terapêutico. As meninas apresentaram redução da sintomatologia e elaboraram crenças mais funcionais em relação à experiência do abuso, o que proporcionou uma melhora na qualidade de vida. Dessa forma, é possível inferir que o modelo de intervenção avaliado foi efetivo na redução de sintomas de depressão, ansiedade e TEPT, bem como na reestruturação de crenças disfuncionais das participantes do estudo. A comparação de tais resultados com um grupo controle permitiria avaliar se a redução da sintomatologia não ocorreu apenas devido à passagem de tempo. Contudo, o delineamento com grupo controle poderia gerar problemas metodológicos, tal como a perda de participantes do grupo controle devido ao tempo de espera, e problemas éticos, pois o estudo não oportunizaria o acesso imediato ao tratamento a crianças e adolescentes em situação de risco. No entanto, alguns estudos confirmam que a passagem do tempo não é responsável pela remissão ou redução de sintomas decorrentes de abuso sexual e que adultos que passaram por essa experiência na infância permanecem com dificuldades psicológicas durante a vida (Cloitre, Cohen, Koenen \& Han., 2002; Edwards \& cols., 2001; Jonzon \& Lindblad, 2004; Lanktree \& Briere, 1995).

\section{Considerações Finais}

A psicologia tem contribuído para a compreensão do abuso sexual infantil, por meio de estudos sobre dinâmica familiar, incidência epidemiológica, conseqüências do trauma para o desenvolvimento e intervenções clínicas. A avaliação da eficácia/efetividade de intervenções psicoterápicas tem sido um importante desafio para psicólogos clínicos e pesquisadores, uma vez que implica articular conhecimentos sobre pesquisa científica e prática clínica (Colin, 1993). Dessa forma, aspectos teóricos, metodológicos, éticos e técnicos devem estar coordenados em pesquisas sobre psicoterapia com o objetivo de produzir avanços no tratamento de problemas psicológicos, contribuindo para a promoção de saúde e qualidade de vida.

A terapia cognitiva-comportamental, desde suas primeiras formulações, tem articulado a pesquisa e a prática clínica (Beck \& Alford, 1997/2000), e a avaliação de modalidades de tratamento tem apontado a eficácia/efetividade das técnicas cognitivas e comportamentais na reestruturação da memória traumática e redução de sintomas de ansiedade, depressão

e transtorno do estresse pós-traumático identificados nas vítimas de abuso sexual. Além disso, como demonstrado neste estudo, o formato grupal tem apresentado resultados superiores no tratamento de crianças e adolescentes que experienciaram abuso sexual (Calhoun \& Resick, 1993/1999; Celano \& cols., 2002; Cohen \& cols., 2005). O modelo adaptado de grupoterapia cognitiva-comportamental proposto por Habigzang e Caminha (2004) reduziu sintomas de depressão, ansiedade e transtorno do estresse pós-traumático, bem como modificou a percepção de diferença em relação aos pares, a culpa pela situação do abuso e pelas modificações na configuração familiar, identificadas na avaliação clínica inicial e durante o processo terapêutico. Outro aspecto positivo identificado foi a melhora no desempenho escolar, embora não testado, mas observado pelas participantes e seus cuidadores, em termos de rendimento e relacionamento interpessoal. Tal melhora pode ser reflexo da redução de sintomas psicológicos, bem como da melhora da auto-estima das participantes. As técnicas empregadas na etapa da psicoeducação foram importantes para a reestruturação de crenças disfuncionais. A psicoeducação e o treino de inoculação do estresse contribuíram para a redução de sintomas de transtorno do estresse pós-traumático, sendo que o jogo sobre a memória e o botão de emergência foram as estratégias que as meninas relataram adotar, quando apresentavam sintomas de revivência do trauma em seu cotidiano. A etapa final, de prevenção à recaída, promoveu a elaboração de estratégias de proteção, por meio da identificação de situações de risco e das oficinas sobre sexualidade e sobre o Estatuto da Criança e do Adolescente. A oficina de psicomotricidade foi vivenciada com intensidade pelas participantes e foi a atividade mais lembrada por elas na avaliação final. Os resultados clínicos deste estudo comprovam a efetividade da aplicação do modelo de Habigzang e Caminha (2004). A aplicação dos instrumentos psicológicos nas diferentes etapas da intervenção e a organização dessas etapas, conforme objetivos e técnicas empregadas, qualificaram o modelo inicial proposto.

Considerando os resultados do processo e o impacto da intervenção cognitivo-comportamental sobre a sintomatologia apresentada pelas participantes, pode-se concluir que foi efetiva e atuou na promoção de resiliência e na melhoria da qualidade de vida das vítimas de violência sexual e suas famílias. O desenvolvimento de novos estudos com um maior número de participantes pode potencializar os resultados encontrados, uma vez que amostras maiores possibilitarão a utilização de estatísticas mais sofisticadas para a análise dos dados e a generalização dos resultados. Além disso, sugere-se que ocorra o acompanhamento durante um ano após o término da intervenção para avaliar a manutenção dos efeitos terapêuticos.

Os resultados do presente estudo estão sendo utilizados para a reformulação do modelo de grupoterapia. Essa refor- 
mulação inclui a redução no número de sessões para melhorar a relação custo/eficiência, bem como a modificação de alguns dispositivos utilizados nas sessões. Tal modificação referese à reformulação da etapa de psicoeducação, reduzindo o número de sessões de sete para cinco. A "caminhada feita em confiança" ocorrerá já na primeira sessão e na segunda será utilizado um documentário sobre abuso sexual. A psicoeducação, quanto ao modelo cognitivo-comportamental, será realizada unindo a noção de pensamentos e reações fisiológicas em uma mesma sessão. A reformulação desse modelo e sua qualificação podem contribuir para o tratamento de crianças e adolescentes vítimas de abuso sexual atendidas em programas públicos de saúde mental, uma vez que a grupoterapia é um modelo replicável, sendo necessária a capacitação dos profissionais. Serviços de saúde pública de alguns municípios do Rio Grande do Sul já foram capacitados e estão buscando multiplicar os resultados deste estudo piloto.

\section{Referências}

Astin, M. C. \& Resick, P. A. (2002). Tratamento cognitivocomportamental do transtorno de estresse pós-traumático. Em V. Caballo (Org.), Manual para o Tratamento Cognitivo Comportamental dos Transtornos Psicológicos (pp.171-210). Porto Alegre: Artes Médicas.

Beck, A. \& Alford, B. A. (2000). O poder integrador da terapia cognitiva (M. C. Monteiro, Trad.). Porto Alegre: Artes Médicas (Trabalho original publicado em 1997)

Biaggio, A. \& Spielberger, C. D. (1983). Inventário de ansiedade traço-estado-Idate-C Manual. Rio de Janeiro: CEPA.

Calhoun, K. S. \& Resick, P. A. (1999). Transtorno do estresse póstraumático. Em D. Barlow (Org.), Manual Clínico dos Transtornos Psicológicos (M. R. B. Osório, Trad.) (pp. 63-118). Porto Alegre: Artes Médicas. (Trabalho original publicado em 1993)

Celano, M. Hazzard, A. Campbell, S. K. \& Lang, C. B. (2002). Attribution retraining with sexually abused children: Review of techniques. Child Maltreatment, 7(1), 64-75.

Cloitre, M., Cohen, L. R., Koenen, K. \& Han, H. (2002). Skills training in affective and interpersonal regulation followed by exposure: A phase-based treatment for PTSD related to childhood abuse. Journal of Consulting and Clinical Psychology, 70(5), 1067-1074.

Cohen, J. A. (2003). Treating acute posttraumatic reactions in children and adolescents. Society of Biologial Psychiatry, 53, 827-833.

Cohen, J. A. \& Mannarino, A. P. (2000). Predictors of treatment outcome in sexually abused children. Child Abuse \& Neglect, 24(7), 983-994.

Cohen, J. A. Mannarino, A. P. \& Knudsen, K. (2005). Treating sexually abused children: One year follow-up of a randomized controlled trial. Child Abuse \& Neglect, 29, 135-145.

Cohen, J. A, Mannarino, A. P. \& Rogal, S. (2001). Treatment practices for childhood posttraumatic stress disorder. Child Abuse \& Neglect, 25, 123-135.

Colin, R. (1993). Real World Research: A resource for social sciences and practioner-reseacher. Oxford: Blackwell.

Deblinger, E., Stauffer, L. B. \& Steer, R. A. (2001). Comparative efficacies of supportive and cognitive behavioral group therapies for young children who have been sexually abused and their nonoffending mothers. Child Maltreatment, 6(4), 332-343.
Del Ben, C. M., Vilela, J. A., Crippa, J.A., Hallak, J. E., Labate, C. M. \& Zuardi, A. W. (2001). Confiabilidade da entrevista estruturada para o DSM-IV - versão clínica traduzida para o português. Revista Brasileira de Psiquiatria, 23(3), 156-159.

Edwards, V. J., Anda, R. F., Nordenberg, D. F, Felitti, V. J, Williamson, D. F. \& Wright, J. A. (2001). Bias assessment for child abuse survey: Factors affecting probability of response to a survey about childhood abuse. Child Abuse \& Neglect, 25, 307-312.

Giacomoni, C. H. (1998). Desempenho acadêmico, controle percebido e eventos de vida como preditores de bem-estar subjetivo em crianças. Dissertação de Mestrado. Universidade Federal do Rio Grande do Sul, Porto Alegre.

Gouveia, V. V., Barbosa, G. A., Almeida, H. J. F. \& Gaião, A. A. (1995). Inventário de depressão infantil - CDI: Estudo de adaptação com escolares de João Pessoa. Jornal Brasileiro de Psiquiatria, 44, 345-349.

Habigzang, L.F. \& Caminha, R.M. (2004). Abuso sexual contra crianças e adolescentes: conceituação e intervenção clínica. São Paulo: Casa do Psicólogo.

Habigzang, L. F., Hatzenberger, R., Dala Corte, F., Stroeher, F. \& Koller, S. H. (2006). Grupoterapia cognitivo-comportamental para meninas vítimas de abuso sexual: Descrição de um modelo de intervenção. Psicologia Clínica,18(2), 163-182.

Habigzang, L. F., Stroeher, F., Dala Corte, F., Hatzenberger, R. \& Koller, S. H. (no prelo). Avaliação psicológica em casos de abuso sexual na infância e adolescência. Psicologia: Reflexão e Crítica.

Heflin, A. H. \& Deblinger, E. (1999). Tratamento de um adolescente sobrevivente de abuso sexual na infância. Em M. Reinecke, F. Dattilio \& A. Freeman (Orgs.), Terapia cognitiva com crianças e adolescentes: manual para a prática clínica (pp.161-178, M. R. Hofmeister, Trad.). Porto Alegre: Artes Médicas. (Trabalho original publicado em 1996)

Hetzel-Riggin, M. D., Brausch, A. M. \& Montgomery, B. S. (2007). A meta-analytic investigation of therapy modality outcomes for sexually abused children and adolescents: An exploratory study. Child Abuse \& Neglect, 31, 125-141.

Jonzon, E. \& Lindblad, F. (2004). Disclosure, reactions and social support: Findings from a sample of adult victims of child sexual abuse. Child Maltreatment, 9(2), 190-200.

Knell, S. M. \& Ruma, C. D. (1999). Terapia do jogo com crianças sexualmente abusadas. Em M. Reinecke, F. Dattilio, \& A. Freeman (Orgs.), Terapia cognitiva com crianças $e$ adolescentes: manual para a prática clínica (pp.277-295, M. R. Hofmeister, Trad.). Porto Alegre: Artes Médicas. (Trabalho original publicado em 1996)

Kovacs, M. (1992). Children's Depression Inventory Manual. Los Angeles: Western Psychological Services.

Kristensen, C. H. (1996). Abuso sexual em meninos. Dissertação de Mestrado, Universidade Federal do Rio Grande do Sul, Porto Alegre.

Kruczek, T. \& Vitanza, S. (1999). Treatment effects with an adolescents abuse survivor's group. Child Abuse \& Neglect, 23(5), 477-485.

Lanktree, C. B. \& Briere, J. (1995). Outcome of therapy for sexually abused children: A repeated measures study. Child Abuse \& Neglect, 19(9), 1145-1155.

Lipp, M. E. \& Lucarelli, M. D. M. (1998). Escala de stress infantil - ESI: Manual. São Paulo: Casa do Psicólogo. 


\section{Intervenção Psicológica em Abuso Sexual}

Mannarino, A. P., Cohen, J. A. \& Berman, S. R. (1994). The children's attributions and perceptions scale: A new measure of sexual abuse-related factors. Journal of Clinical Child Psychology, 23(2), 204-211.

Manual Diagnóstico e Estatístico dos Transtornos Mentais (2002). (4 ed. Revisada). Porto Alegre: Artes Médicas.

McCrone, P., Weeramanthri,T., Knapp, M., Rushton, A., Trowell, J., Miles, G. \& Kolvin, I. (2005). Cost-effectiveness of individual versus group psychotherapy for sexually abused girls. Child and Adolescent Mental Health, 10(1), 26-31.

McGain, B. \& McKinzey, R. K. (1995). The efficacy of group treatment in sexually abused girls. Child Abuse \& Neglect, 19(9), 1157-1169.

Osofsky, J. D. (1995). The effects of exposure to violence on young children. American Psychologist, 50(9), 782-788.

Runyon, M. K., \& Kenny, M. C. (2002). Relationship of atribucional style, depression and post trauma distress among children who suffered physical or sexual abuse. Child Maltreatment, 7(3), 254-264.

Saywitz, K. J., Mannarino, A. P., Berliner, L. \& Cohen, J. A. (2000). Treatment for sexually abused children and adolescents. American Psychologist, 55(9), 1040-1049.
Smith, E. R. (1996). Psicoterapia de grupo com crianças sexualmente abusadas. EmH. I. Kaplan \& B. J. Sadock (Orgs.), Compêndio de psicoterapia de grupo (pp.441-457, J. O. A. Abreu \& D. Batista, Trad.). Porto Alegre: Artes Médicas. (Trabalho original publicado em 1993)

The Metropolitan Toronto Special Committee on Child Abuse (1995). Child Sexual Abuse Protocol (3rd. ed.). Toronto: Author.

Valle, A. L. \& Silovsky, J. F. (2002). Attributions and adjustment following child sexual and physical abuse. Child Maltreatment, 7(1), 9-24.

Vera, M. N. \& Vila, J. (1996). Técnicas de relaxamento. Em V. E. Caballo (Org.), Manual de Técnicas de Terapia e Modificação do Comportamento (pp. 147-166). São Paulo: Santos.

Recebido em 15.02.2007

Primeira decisão editorial em 27.06.2007

Versão final em 10.09.2007

Aceito em 01.12.2007 